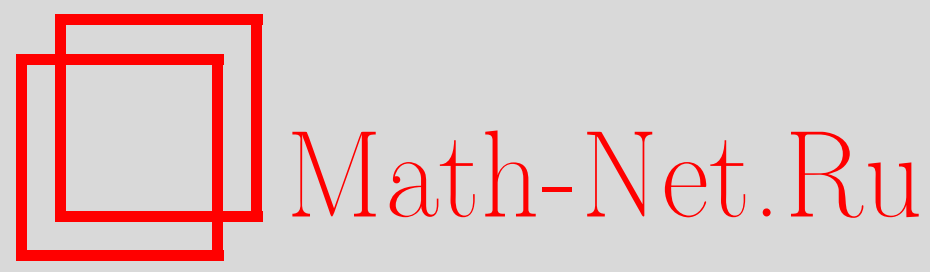

Г. В. Коваль, Об асимптотическом пределе матричных элементов канонического оператора для комплексного ростка в точке, Матем. заметки, 1998, том 63, выпуск 3, 479-480

DOI: https://doi.org/10.4213/mzm1307

Использование Общероссийского математического портала Math-Net.Ru подразумевает, что вы прочитали и согласны с пользовательским соглашением http://www . mathnet.ru/rus/agreement

Параметры загрузки:

IP : 3.95 .254 .165

26 апреля 2023 г., 13:20:26 


\section{ОБ АСИМПТОТИЧЕСКОМ ПРЕДЕЛЕ МАТРИЧНЫХ ЭЛЕМЕНТОВ КАНОНИЧЕСКОГО ОПЕРАТОРА ДЛЯ КОМПЛЕКСНОГО РОСТКА В ТОЧКЕ}

\section{Г.В. Коваль}

Рассмотрим функции из $L^{2}\left(\mathbb{R}^{n}\right)$, соответствующие комплексному ростку [1] в точке:

$$
\psi_{\nu}(x)=N_{\nu} e^{i S(x) / h} \bar{\Lambda}_{1}^{\nu_{1}} \cdots \bar{\Lambda}_{n}^{\nu_{n}} 1,
$$

где $\nu=\left(\nu_{1}, \ldots, \nu_{n}\right)$ - мультииндекс, $N_{\nu}$ - нормировочная константа, действие $S(x)$ имеет вид

$$
S(x)=\left\langle p_{0}, x-x_{0}\right\rangle+\frac{1}{2}\left\langle x-x_{0}, B C^{-1}\left(x-x_{0}\right)\right\rangle,
$$

а дифференциальные операторы $\bar{\Lambda}_{j}$ выражаются следующим образом:

$$
\bar{\Lambda}_{j}=\frac{\sqrt{h}}{i}\left\langle\bar{z}_{j}, \frac{\partial}{\partial x}\right\rangle-\frac{1}{\sqrt{h}}\left\langle\bar{w}_{j}-B C^{-1} \bar{z}_{j}, x-x_{0}\right\rangle, \quad j=\overline{1, n} .
$$

Здесь матрицы $B$ и $C$ построены из $n$-мерных столбцов $w_{i}, z_{i}, i=\overline{1, n}$, соответственно, которые удовлетворяют условиям

$$
\left\langle w_{i}, z_{j}\right\rangle-\left\langle w_{j}, z_{i}\right\rangle=0,\left\langle w_{i}, \bar{z}_{j}\right\rangle-\left\langle\bar{w}_{j}, z_{i}\right\rangle=2 i \delta_{i j}, \quad i, j=\overline{1, n} .
$$

Функции вида (1) при $h \rightarrow 0$ являются приближенными собственньпи функциями уравнений на собственные значения в различных задачах, в том числе в задачах квантовой теории поля и задачах о большом числе тождественных бозонов [2], когда размерность пространства $n$ бесконечна. В этих случаях в качестве $z_{i}, w_{i}, i=\overline{1, n}$, берутся решения соответствуюшей данной задаче системы в вариациях [1]. Пусть $L(p, x) \in C_{0}^{\infty}\left(\mathbb{R}^{2 n}\right)$ есть символ $h$-псевдодифференциального оператора (см. [3]), $\widehat{L}=L\left(\hat{\widehat{p}}, \frac{1}{\widehat{x}}\right)$. Тогда для матричных элементов оператора $\widehat{L}$ на функциях (1),

$$
\left(\psi_{\mu}, \widehat{L} \psi_{\nu}\right)=\iint d x d p L(p, x) \psi_{\nu}(x) \widetilde{\psi}_{\mu}^{*}(p) \frac{e^{-\frac{i}{h}\langle p, x\rangle}}{(2 \pi h)^{n / 2}}
$$

можно найти предел при $h \rightarrow 0$. Именно, легко показать, что

$$
\begin{aligned}
& \lim _{h \rightarrow 0} \frac{1}{h^{|\mu-\nu| / 2}} \iint d p d x L(p, x) \psi_{\nu}(x) \widetilde{\psi}_{\mu}^{*}(p) \frac{e^{-\frac{i}{h}\langle p, x\rangle}}{(2 \pi h)^{n / 2}} \\
& \quad=\left.\prod_{i=1}^{n} \alpha_{i}\left(\nu_{i}, \mu_{i}\right)\left(A_{i}\left(\nu_{i}, \mu_{i}\right)\right)^{\left|\mu_{i}-\nu_{i}\right|} F(x, p)\right|_{(x, p)=\left(x_{0}, p_{0}\right)},
\end{aligned}
$$

где константы $\alpha_{i}\left(\nu_{i}, \mu_{i}\right)$ имеют вид

$$
\alpha_{i}\left(\nu_{i}, \mu_{i}\right)=\left(\frac{1}{i \sqrt{2}}\right)^{\left|\mu_{i}-\nu_{i}\right|} \frac{1}{\left|\mu_{i}-\nu_{i}\right| !}\left(\sqrt{\frac{\mu_{i} !}{\nu_{i} !}}\right)^{\operatorname{sign}\left(\mu_{i}-\nu_{i}\right)}
$$

Работа выполнена при финансовой поддержке объединенного проекта фонда INTAS и Российского фонда фундаментальных исследований, грант № 95-91. 
операторы $A_{i}\left(\nu_{i}, \mu_{i}\right)$ выражаются следуюшим образом:

$$
A_{i}\left(\nu_{i}, \mu_{i}\right)= \begin{cases}1, & \nu_{i}=\mu_{i} \\ \left\langle z_{i}, \frac{\partial}{\partial x}\right\rangle+\left\langle w_{i}, \frac{\partial}{\partial p}\right\rangle, & \nu_{i}<\mu_{i} \\ \left\langle\bar{z}_{i}, \frac{\partial}{\partial x}\right\rangle+\left\langle\bar{w}_{i}, \frac{\partial}{\partial p}\right\rangle, & \mu_{i}<\nu_{i}\end{cases}
$$

и $|\mu-\nu|=\sum_{i=1}^{n}\left|\mu_{i}-\nu_{i}\right|$. В частности, в задаче для $n$-мерного осциллятора функции (1) являются точными решениями уравнения Шрёдингера. Из (2) видно, что функции Вигнера

$$
\rho_{\nu \mu}(p, x)=\frac{1}{h^{|\mu-\nu| / 2}} \psi_{\nu}(x) \widetilde{\psi}_{\mu}^{*}(p) \frac{e^{-\frac{i}{h}\langle p, x\rangle}}{(2 \pi h)^{n / 2}}
$$

при $h \rightarrow 0$ сходятся в слабом смысле к обобщенным функциям

$$
\lim _{h \rightarrow 0} \rho_{\nu \mu}(p, x)=(-1)^{|\mu-\nu|} \prod_{i=1}^{n} \alpha_{i}\left(\nu_{i}, \mu_{i}\right)\left(A_{i}\left(\nu_{i}, \mu_{i}\right)\right)^{\left|\mu_{i}-\nu_{i}\right|} \delta(p) \delta(x),
$$

где операторы $A_{i}\left(\nu_{i}, \mu_{i}\right)$ даются формулой $(3)$, в которой в качестве $z_{i}, w_{i}$ взяты решения соответствующей системы в вариациях [1]. Так как функции Вигнера при $h \rightarrow 0$ сходятся в слабом смыслек собственньт функциям оператора Лиувилля, функции (4), очевидно, являются обобщенньми собственными функциями оператора Лиувилля для осциллятора. В этом смысле операторы (3) можно назвать операторами рождения для уравнения Лиувилля, поскольку собственные значения оператора Лиувилля, соответствующие (4), суть

$$
\lambda_{\nu \mu}=\sum_{i=1}^{n} \beta_{i}\left(\nu_{i}-\mu_{i}\right)
$$

где $\beta_{i}-$ собственные значения системы в вариациях [1]. Отметим также, что в одномерном случае обобщенная функция

$$
\rho_{k}(p, x)=\frac{1}{\varepsilon^{|k| / 2}} \delta\left(\frac{x^{2}+p^{2}}{2}-\varepsilon\right) e^{i k \varphi},
$$

где $k$-целое число, а $\varphi$ - угловая переменная, является собственной функцией оператора Лиувилля на уровне энергии $\varepsilon$ и слабо сходится при $\varepsilon \rightarrow 0$ к функциям вида (4).

\section{СПИСОК ЦИТИРОВАННОЙ ЛИТЕРАТУРЫ}

1. Маслов В. П. Комплексный метод ВКБ в нелинейных уравнениях. М.: Наука, 1977. 2. Маслов В. П., Шведов О. Ю. // ТМФ. 1995. Т. 104. № 3. С. 479-506. 3. Маслов В. П., Федорюк М. В. Квазиклассическое приближение для уравнений квантовой механики. М.: Наука, 1976.

Московский государственный университет им. М.В.Ломоносова 